\title{
Control and optimal management of a heliostat field for solar power tower systems
}

\author{
Nicolás Calvo Cruz \\ Dept. of Informatics, ceiA3 \\ University of Almería \\ Almería, Spain \\ ncalvocruz@ual.es \\ Manuel Berenguel \\ Dept. of Informatics, CIESOL-ceiA3 \\ University of Almería \\ Almería, Spain \\ beren@ual.es
}

\author{
José Domingo Álvarez \\ Dept. of Informatics, CIESOL-ceiA3 \\ University of Almería \\ Almería, Spain \\ jhervas@ual.es
}

\author{
Juana López Redondo \\ Dept. of Informatics, ceiA3 \\ University of Almería \\ Almería, Spain \\ jlredondo@ual.es
}

\author{
Pilar Martínez Ortigosa \\ Dept. of Informatics, ceiA3 \\ University of Almería \\ Almería, Spain \\ ortigosa@ual.es
}

\author{
Ryszard Klempous \\ Dept. of Electronics \\ Wroctaw University of Science and Technology \\ Wrocław, Poland \\ ryszard.klempous@pwr.edu.pl.
}

\begin{abstract}
The competitiveness of Concentrated Solar Power (CSP) plants over conventional ones still has to be improved. For CSP systems based on Central Receivers (CRS), one of the challenges to face is the optimal management of the aim points of the heliostats which form the collector field. The flux distribution that the field projects on the receiver must be carefully controlled to get an adequate form and to avoid dangerous flux peaks that might damage the receiver. Phenomena such as cloud transients can result in pronounced temperature gradients that reduce the life expectancy of receivers. Therefore, it is necessary to develop a control system which ensures that the critical parameters of the receiver (e.g., temperatures, solar radiation, pressure, mass flow) are always within the allowed range. This work presents an automatic control system connected to an optimization method based on a genetic algorithm which theoretically configures the field to obtain any desired flux distribution. It is a heuristic feedback controller that minimizes the error between the flux distribution theoretically computed and that obtained over time. The control logic tries to reduce the effect of perturbations as well as modeling and optimization errors that might have affected the genetic optimizer when computing the initial operating state.
\end{abstract}

Index Terms-solar tower systems, heliostat field, flux distribution

\section{INTRODUCTION}

The awareness of pollution and depletion problems of traditional fuel-based electricity production has resulted in a growing interest in renewable energy [1], [2]. For this reason, there is great interest in renewable sources and technologies. Among them, concentrating solar power (CSP) systems are especially attractive because of their hybridization possibilities and their output stability through thermal storage [3]. This work focuses on solar power tower systems (SPT), which belong to the group of CSP technologies and are popular because of providing high thermodynamic efficiency and their high scalability [3], [4].

In general, SPT plants are composed of multiple highly reflectant mirrors known as heliostats, a radiation receiver, a power block and thermal storage. Heliostats have orientable structures to track the movement of the sun while reflecting the incident solar radiation on the receiver, which is commonly on top of a tower. There is a heat-transfer fluid that flows inside the receiver to be heated. Once it is hot enough, this fluid can be used in a turbine cycle to produce electricity. It can also be stored to operate the plant with reduced light conditions. Figure 1 includes a simple but descriptive depiction of this kind of power plant. The interested reader can find further information about SPT in [5], [6].

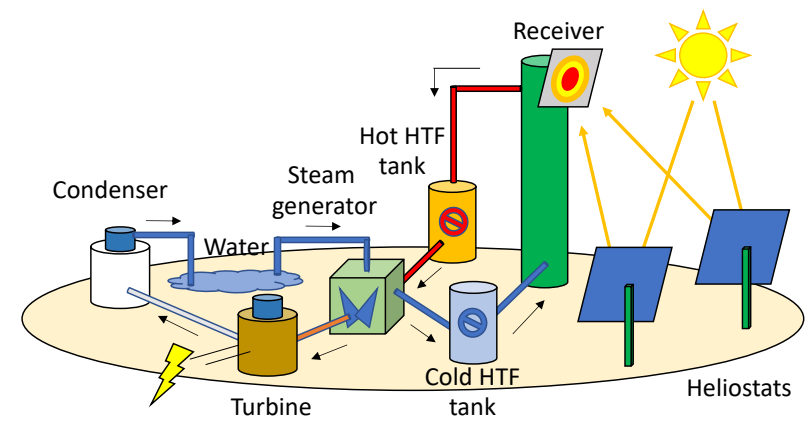

Fig. 1. Simplified scheme of a CRS plant

The radiation receiver in an SPT plant is a critical and expensive component, and its conservation is relevant to reduce the cost of produced electricity [7]. Unfortunately, the flux distribution that the heliostat field projects on the receiver can generate dangerous radiation peaks and temperature gradients that may damage it [7], [8]. Moreover, the achieved flux distribution also affects the production efficiency of the plant since it determines the interception factor, i.e., the ratio of spilled radiation [4], [8]. Therefore, it is necessary to control the flux distribution that the heliostat field projects on the receiver to keep it safe while properly profiting the incoming power [9], [10]. 
Controlling the heliostat field of an SPT plant encompasses from selecting the set of active heliostats to defining their aim points to keep the desired flux profile at any time [11]. Not only the apparent solar movement and variations in direct normal irradiance must be considered but also modeling and physical errors as well as cloud transients. In this context, human-based decisions have limited applicability, and there exist multiple proposals to deal with controlling heliostat fields with different goals and scopes [10], [12].

In [7], the authors adapt the traditional TABU search algorithm for combinatorial optimization [13] to find the best aim point out of a discrete set for each heliostat. The method looks for a homogeneous flux distribution while also trying to achieve low spillage rates. It is used to define an open-loop controller based on analytical flux map simulations. In [8], the authors also design an optimizer to achieve homogeneous flux maps with acceptable spillage values, but they opt for adapting a genetic algorithm [14] in a similar combinatorial approach. Fields are also analytically simulated. In [15], a combination of the two previous strategies is proposed and successfully tested: the TABU search generates good initial solutions for the genetic procedure. The behavior of heliostats is analytically modeled but after fitting accurate records. In [16], the aim is to obtain a uniform flux distribution with low spillage too. An ad-hoc TABU combinatorial optimizer is developed. In [17], adapts the principles of ant colony optimization [18] to assigning the best point to each heliostat in a combinatorial context. Although dangerous radiation peaks are avoided, the focus is on maximizing the performance of the receiver. Besides, accurate ray-tracing simulations are used to model the field. In [9], the authors seek uniform flux shapes while also minimizing spillage and keeping a minimum level of energy. The approach is still combinatorial with analytical flux estimation, but an integer programming solver is applied with almost real-time compatibility. In [19], homogeneity and high power exploitation are intended but an interior-point method is used to solve the continuous non-linear optimization problem. Finally, in [10], the authors design a general solution for the optimization problem which covers from heliostat selection to aiming in a continuous, i.e., not combinatorial, search space. Besides, the goal is not fixed but oriented to replicate any desired flux profile, and a new pseudo-analytical model is used. This work extends that one to form a complete controller.

The rest of the paper is structured as follows: Section II explains the base aiming point optimization strategy. Section III describes the heuristic control strategy proposed. Section IV shows some preliminary results obtained through the method presented herein. Finally, Section V draws the main conclusions and presents the future work.

\section{OptimizATION OF THE AIMING STRATEGY}

The current problem focuses on replicating a given flow distribution in a receiver. To this aim, a subset of heliostats in the field, as well as their aiming points, must be selected. An optimization problem can then be defined based on minimizing the difference between the desired flux distribution and the achieved one. The complete definition of the optimization problem appears in [10]. We encourage the interested reader to examine it.

To deal with this complex optimization problem, a two-layer algorithm has been developed. The first stage is responsible for finding a good subset of heliostats and allocating an initial aim point to every selected heliostat. Withoug going into details, this layer follows the structure of a classic Genetic Algorithm (GA), i.e. it initially generates a random initial population and then executes a main loop. Each loop iteration consists of a sequence of procedure calls for (i) parents selection, (ii) reproduction, (iii) spring mutation and (iv) replacement. At the end of the GA, the best individual found is provided as the solution.

To select the progenitors, the stochastic tournament-based method has been considered [14], [20]. The implemented reproduction method has been the 'Uniform' crossover technique described in [14]. Concerning the mutation of the offspring, it is based on randomly activating and deactivating heliostats on them.

Finally, an elitist replacement has been used, where the best individuals are directly chosen, regardless of whether its origin is the initial population or the descendants.

The second layer tries to improve each aim point assignment by using a Gradient-Search (GS) method. Notice that the heliostat selection is not further modified, only the aim points of the active heliostats are changed to minimize the objective function.

Further details about the complete optimization method are provided in [10].

\section{DEVELOPMENT OF A HEURISTIC CONTROL STRATEGY}

The optimization aiming strategy described in the previous section works with a model of the heliostat field, thus, the optimization process is model-driven but, unfortunately, a perfect modeling is a utopia and it is common that modeling errors appear. It is necessary to translate the optimization results to reality, correct modeling errors, and keep the desired flux map up over time as well as in time despite disturbances. This is the mission of the control system.

The heuristic control strategy developed in this work is applied after the optimization one and it uses, as starting point, the heliostats subset and the aiming points that the optimization procedure has calculated. The control strategy has three main actions to obtain the desired flux distribution. These actions are the following:

1) Activating heliostats that are not in the initial subset calculated by the optimization aiming strategy. This action is taken when the total incident power on the receiver is less than the total power of the setpoint. Thus, one or more heliostats must be focused on the receiver. It (or they) will be aimed at the lowest peak of the difference between the setpoint and the current incident flux onto the receiver.

2) Deactivating heliostats that, initially, are in the initial subset. This is the opposite case that the previous one, 
that is, there is more incident power on the receiver than the total one in the setpoint. Therefore, the active heliostat (or heliostats) whose incident power is the nearest to the total difference between the setpoint and the current incident flux onto the receiver, without exceeding, is deactivated.

3) Re-aiming heliostats. In this case, the difference, or error, between the setpoint and the current incident flux onto the receiver in each point of the receiver is calculated and, later, the maximum and minimum of these differences are selected. The maximum difference means that there is too much power in this point and, on the opposite, the minimum difference means that there is less power in this point that the necessary one. Thus, the control sytem looks for the closest heliostat to the maximum difference point and moves it to the minimum difference one.

\section{RESUlts}

In this section the results of the aiming strategy and the heuristic control strategy are presented. To this aim, a virtual CRS plant is built in the open-source ray-tracer Tonatiuh [21]. The heliostat field is composed of 541 heliostats with 12 facets and a total surface close to $40 \mathrm{~m}^{2}$. On the other hand, the receiver is a flat one, that is placed on the top of a tower 155 $\mathrm{m}$ above the ground, with a surface of $10 \times 10 \mathrm{~m}$. The layout of the heliostat field is depicted in Fig. 2

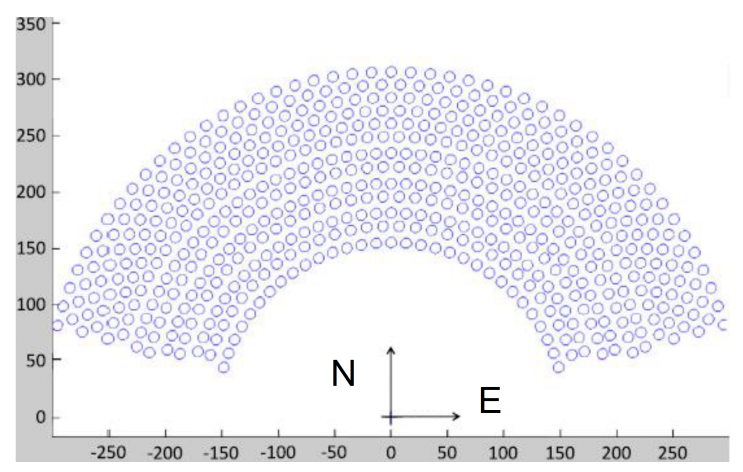

Fig. 2. Heliostat field layout built in Tonatiuh

The control system must reach as setpoint a homogeneous flux distribution of $80 \mathrm{~kW} / \mathrm{m}^{2}$, as can be seen in Fig. 3(a) First, the optimization aiming strategy, that is explained in Section II, is applied. Thus, a subset of 173 heliostats is selected and aimed to the receiver. The incident flux distribution reached once the optimization aiming strategy is finished is showed in Fig. 3(b) with a standard deviation from the setpoint of $2.8757 \mathrm{~kW} / \mathrm{m}^{2}$. After that, the heuristic control strategy, described in Section III, is applied to this heliostats subset, the results are improved, see Figs. 3(c) and 3(d), and the standard deviation is reduced to $2.3104 \mathrm{~kW} / \mathrm{m}^{2}$. Thus, it is important to highlight that the combination of the optimization aiming strategy together the heuristic control strategy is able to reach any kind of flux distribution reference.

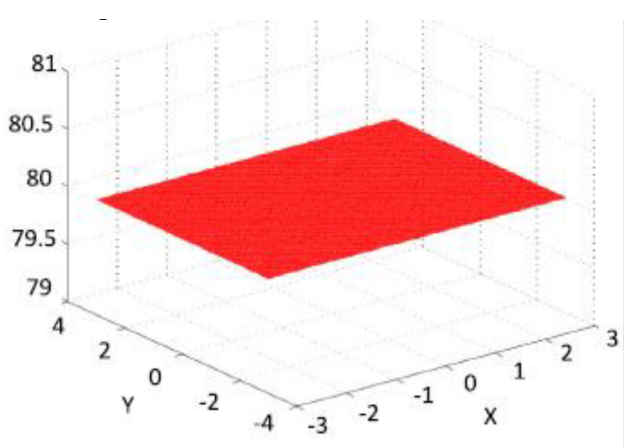

(a) Homogeneous flux distribution reference

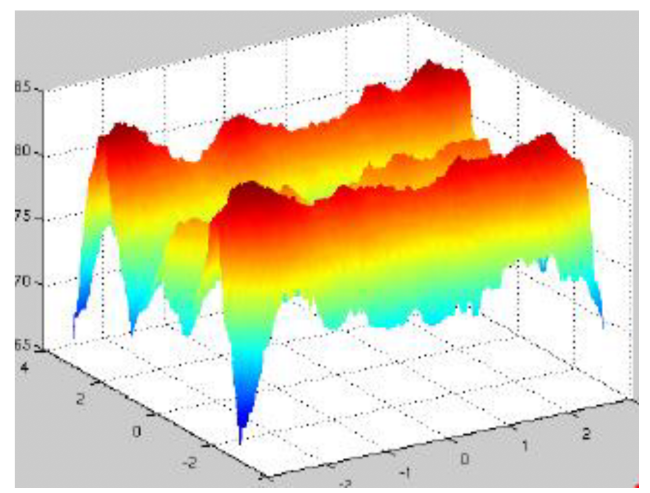

(b) First step. Optimization aiming strategy

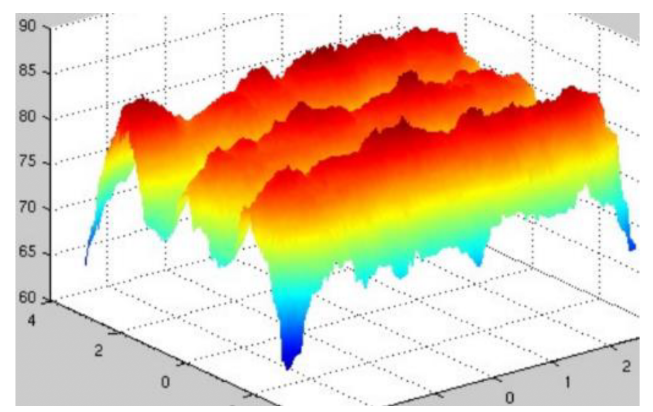

(c) Second step. Heuristic control strategy

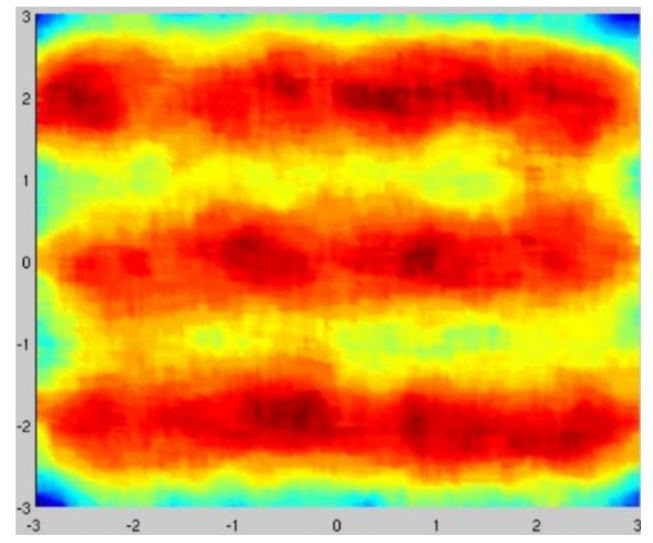

(d) Second step. Heuristic control strategy - Front view

Fig. 3. Results of applying the optimization aiming strategy and the heuristic control system

\section{CONCLUSIONS}

This work presents a heuristic control system to obtain the desired flux distribution for a solar power tower plant. 
The control system is a second step that is used once an optimization aiming strategy is applied. The optimization aiming strategy is devoted to select a subset of heliostats and their aiming points in the receiver to obtain the desired flux distribution onto it. Later, the heuristic control strategy is able to improve the results against model errors. To do this, the heuristic control strategy has three main actions: i) activating new heliostats, ii) deactivating heliostats that, currently, are focused to the receiver and, iii) re-aiming heliostats from hot spots to cold ones. The results presented in the previous section show how the combination of both strategies, optimization and control, is able to obtain any desired flux distribution. Future works are focused to test theses strategies in a real solar power tower plant.

\section{ACKNOWLEDGMENT}

This work has been funded by grants from the Spanish Ministry of Science and Innovation (TIN2015-66680-C2-1$\mathrm{R}$ and CHROMAE DPI2017-85007-R). Nicolás Calvo Cruz (FPU14/01728) is supported by an FPU Fellowship from the Spanish Ministry of Education.

\section{REFERENCES}

[1] S. Kiwan and S. Al Hamad, "On analyzing the optical performance of solar central tower systems on hillsides using biomimetic spiral distribution," Journal of Solar Energy Engineering, vol. 141, no. 1, pp. $1-12,2019$.

[2] J. Wang, L. Duan, and Y. Yang, "An improvement crossover operation method in genetic algorithm and spatial optimization of heliostat field," Energy, vol. 155, pp. 15-28, 2018.

[3] M. Saghafifar, M. Gadalla, and K. Mohammadi, "Thermo-economic analysis and optimization of heliostat fields using aineh code: Analysis of implementation of non-equal heliostats (aineh)," Renewable Energy, 2018.

[4] F. J. Collado and J. Guallar, "A review of optimized design layouts for solar power tower plants with campo code," Renewable and Sustainable Energy Reviews, vol. 20, pp. 142-154, 2013.

[5] S. Alexopoulos and B. Hoffschmidt, "Advances in solar tower technology," WIREs Energy and Environment, vol. 6, no. 1, pp. 1-19, 2017. [Online]. Available: http://dx.doi.org/10.1002/wene.217

[6] D. Y. Goswami, Principles of Solar Engineering, 3rd ed. Taylor \& Francis, 2015.

[7] A. Salomé, F. Chhel, G. Flamant, A. Ferrière, and F. Thiery, "Control of the flux distribution on a solar tower receiver using an optimized aiming point strategy: Application to themis solar tower," Solar Energy, vol. 94, pp. 352-366, 2013.

[8] S. M. Besarati, D. Y. Goswami, and E. K. Stefanakos, "Optimal heliostat aiming strategy for uniform distribution of heat flux on the receiver of a solar power tower plant," Energy Conversion and Management, vol. 84, pp. 234-243, 2014.

[9] T. Ashley, E. Carrizosa, and E. Fernández-Cara, "Optimisation of aiming strategies in solar power tower plants," Energy, vol. 137, pp. 285-291, 2017.

[10] N. C. Cruz, J. D. Álvarez, J. L. Redondo, M. Berenguel, and P. M. Ortigosa, "A two-layered solution for automatic heliostat aiming," Engineering Applications of Artificial Intelligence, vol. 72, pp. 253-266, 2018.

[11] E. F. Camacho, M. Berenguel, and F. R. Rubio, Advanced control of solar plants. Springer Berlin, 1997.

[12] A. Grobler and P. Gauché, "A review of aiming strategies for central receivers," in Proceedings of the second Southern African Solar Energy Conference, 2014.

[13] F. Glover and M. Laguna, "Tabu search," in Handbook of Combinatorial Optimization. Springer, 1998, pp. 2093-2229.

[14] J. L. Redondo, "Solving competitive location problems via memetic algorithms. high performance computing approaches." Ph.D. dissertation, University of Almería, 2009.
[15] A. Grobler, "Aiming strategies for small central receiver systems," Ph.D. dissertation, Stellenbosch: Stellenbosch University, 2015.

[16] Q. Yu, Z. Wang, and E. Xu, "Analysis and improvement of solar flux distribution inside a cavity receiver based on multi-focal points of heliostat field," Applied energy, vol. 136, pp. 417-430, 2014.

[17] B. Belhomme, R. Pitz-Paal, and P. Schwarzbözl, "Optimization of heliostat aim point selection for central receiver systems based on the ant colony optimization metaheuristic," Journal of Solar Energy Engineering, vol. 136, no. 1, p. 011005, 2014.

[18] M. Dorigo and G. Di Caro, "Ant colony optimization: a new metaheuristic," in Proceedings of the 1999 Congress on Evolutionary Computation-CEC99, vol. 2. IEEE, 1999, pp. 1470-1477.

[19] A. J. Gallego, F. Fele, and E. F. Camacho, "On the optimization of irradiance distribution in solar tower plants with flat receivers," in Control Conference (ECC), 2014 European. IEEE, 2014, pp. 24962501.

[20] D. E. Goldberg and K. Deb, "A comparative analysis of selection schemes used in genetic algorithms," in Foundations of Genetic Algorithms. Elsevier, 1991, vol. 1, pp. 69-93.

[21] M. J. Blanco, J. M. Amieva, and A. Mancillas, "The tonatiuh software development project: An open source approach to the simulation of solar concentrating systems," in ASME 2005 International Mechanical Engineering Congress and Exposition. American Society of Mechanical Engineers, 2005, pp. 157-164. 